\title{
Ex Vivo Normothermic Hemoperfusion of the Canine Pancreas: Applications and Limitations of a Modified Experimental Preparation
}

\author{
Frederic Eckhauser, M.D., J. A. Knol, M.D., V. Porter-Fink, M.A., D. Lockery, \\ Leslie Edgcomb, M.D., W. E. Strodel, M.D., David Webb, M.A., \\ AND JERRY SIMMONS, M.D.
}
Departments of Surgery and Pathology Veterans Administration Medical Center, Ann Arbor, Michigan 48105, and University of Michigan Medical School, Ann Arbor, Michigan 48109

Submitted for publication June 23, 1980

\begin{abstract}
Ex vivo recirculation perfusion preparations of the canine pancreas are useful for the study of pancreatic physiology and exocrine secretory function. Vagaries of isolated ex vivo organ perfusion, however, require a thorough familiarity with available circulation media and component technology. Electrolyte and acid-base homeostasis can be facilitated by incorporating a dialysis unit into the perfusion circuit. Viability of the preparation is best assessed by constancy of vascular resistance and oxygen extraction during the period of perfusion. Limitations imposed by this closed method of organ perfusion on studies of pancreatic endocrine function and on duodenopancreatic hormonal interactions (enteroinsular axis) must be recognized.
\end{abstract}

\section{INTRODUCTION}

Perfusion of the isolated canine pancreas was described first by Babkin and Starling in 1926 to study the effects of exogenous secretin on pancreatic blood flow [3]. Since that time, isolated pancreas perfusion has been accomplished successfully in several additional species, including rat [11], rabbit [26], and cat [4]. Denervated pancreaticoduodenal preparations have been used extensively to investigate physiologic exocrine responses to luminal and bloodborne stimuli $[4,14,20]$. More recently, these preparations have been used to elucidate the mechanisms by which intestinal hormones or trophic factors mediate endocrine functions of the pancreatic islets [6-9, 11]. Abnormal enteroinsular regulation of islet function has been studied in a variety of pathologic conditions including celiac disease [6], primary pancreatic disease [7], diabetes mellitus [8], and obesity [9]. The complex paracrine interaction between intestinal and pancreatic hormones is basic to our understanding of nutrient metabolism. In addition, ex vivo pancreatic perfusion in preparation for whole organ or islet cell transplantation has been described [28].

The literature is replete with articles and books describing the technical and mechanical aspects of isolated pancreas perfusion $[14,20,22,24]$. A variety of perfusion media have likewise been described [24]. Successful perfusion is often fraught with considerable difficulties that for some reason are alluded to but rarely discussed completely. Recirculation perfusion, modified by incorporation of a dialysis unit in the perfusion circuit, appears to facilitate electrolyte and acid-base homeostasis and further provides a mechanism to eliminate circulating free hemoglobin and lactic acid from the system. The purpose of this investigation was to develop isolation and perfusion techniques necessary to ensure a viable ex vivo pancreatic perfusion preparation.

\section{MATERIALS AND METHODS}

\section{The Perfusion Apparatus}

The perfusion apparatus illustrated in Fig. 1 is similar to that described by Ritchie and 


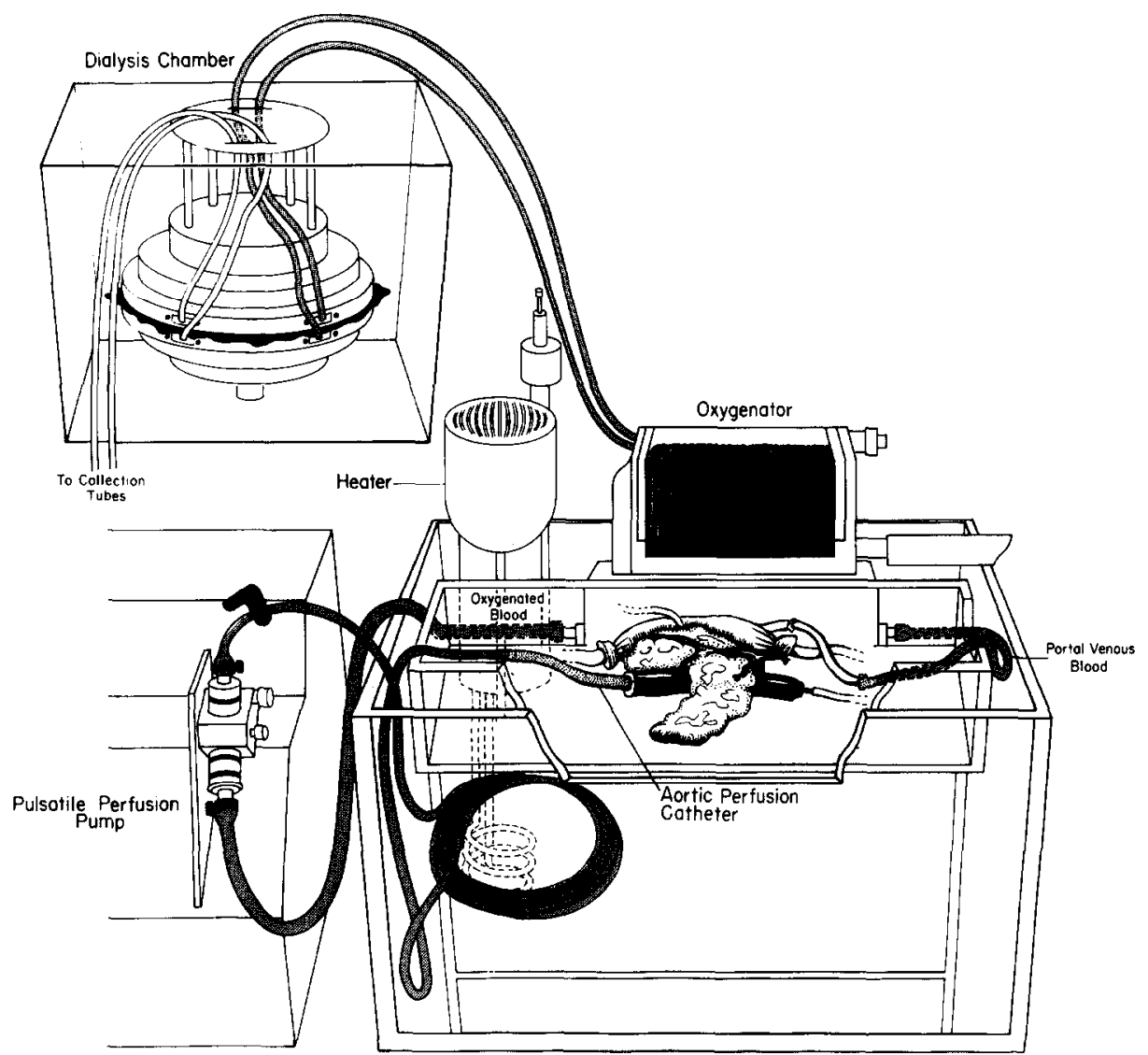

FIG. 1. Illustration of modified organ perfusion apparatus including the the inline dialysis unit.

Hardcastle [22]. The heating chamber is constructed of $1-\mathrm{cm}$ thick Plexiglas and is 37 $\times 32 \times 21 \mathrm{~cm}$. The water bath is filled with 24 liters of tap water which is heated and circulated with a 750-W Braun Thermomix-2.

The organ preparation rests on a polyethylene sheet (Saranwrap) which is stretched over a $28 \times 11-\mathrm{cm}$ Plexiglas frame. This size has proved adequate to support preparations from dogs in the 20 - to $35-\mathrm{kg}$ weight range. The frame fits snugly into a rectangular Plexiglas support which, when fully assembled, supports the entire organ. Indentations are cut at opposite ends of the frame to facilitate placement and securing of arterial and venous perfusion tubing as well as pancreatic duct tubing and pressure monitoring cannulas. A Plexiglas chamber cover is fitted over the support to prevent surface drying of the organ. The Plexiglas cover should be transparent as underperfusion of the isolated organ can be detected by the appearance of blue surface mottling. In its final configuration, the organ rests on the polyethylene sheet which in turn rests directly on the water's surface. Constant mixing of the water provided by the heatermixer is felt to provide an agitation-type action which prevents localized restriction of blood flow through the organ.

A combination oxygenator-reservoir is also suspended in the water bath. This consists of a modified rotating disk oxygenator (Sarnes Company Inc., Ann Arbor, Mich.) with an $800-\mathrm{ml}$ capacity. Throughout the majority of our studies, a $300-\mathrm{ml}$ bloodperfusate mixture was used. Storage of autologous blood in the oxygenator-reservoir eliminates stasis problems and the 300 - 
$\mathrm{ml}$ volume used is generally sufficient to contact the oxygenator disks properly. A gas mixture of $95 \%$ oxygen and $5 \%$ carbon dioxide is passed through a humidifier and delivered to the disk oxygenator. The humidifier consists of a 1.0-liter Erlenmeyer flask with a side arm. Gases are introduced into the apparatus and bubbled through an aquarium air stone. Once humidified, the gas mixture is routed through the side arm to the oxygenator-reservoir. The purpose of the in-line humidifier is to minimize surface drying of the organ preparation.

\section{The Circuit}

Oxygenated blood from the reservoir is circulated with a pulsatile infusion pump (Harvard apparatus, Model 1405). Blood is directed from the perfusion pump through a warming coil which contains an injection sampling port. It then passes through a blood filter (Abbott Y-type blood pump set No. 1746) to the arterial cannula. The rate of perfusion is varied according to the oxygen extraction of the organ but in general remains constant at $20 \pm 5 \mathrm{ml} / \mathrm{min}$.

Polyethylene tubing between the recirculation pump and the arterial cannula is $\mathbf{0 . 3}$ $\mathrm{cm}$ i.d. The tubing from the reservoir to the pump is $0.6 \mathrm{~cm}$ i.d. and $45 \mathrm{~cm}$ in length. The venous tubing consists of $0.5 \mathrm{~cm}$ i.d. polyethylene tubing. This tubing as well as the arterial tubing contains an injection-sampling port. Total priming volume for the entire perfusion circuit including the reservoir is approximately $400 \mathrm{ml}$.

\section{The Dialysis Unit}

An in-line dialysis unit is used to prevent accumulation of potentially deleterious metabolic by-products and to prevent progressive hypernatremia due to dehydration. A similar system was described previously by Long and Lyons in 1954 [16] and later modified by Richardson and Ulfelder in 1960 [21] and by Abraham et al. in 1968 [1].

The dialysis unit was modified from a
Technicon Model 1 dialyzer salvaged from a creatinine analyzer. Early attempts at formal dialysis proved largely unsuccessful due to the small size of the apparatus compared with the large metabolic demands of the organ. In its final, usable form, dialysis is actually a form of selective ultrafiltration. A Technicon autoanalyzer proportioning pump is used to draw blood from the oxygenator-reservoir at a rate of $6 \mathrm{ml} /$ min. Perfusate without red cells is drawn simultaneously at the same rate from another reservoir. The two channels are joined and the admixture is run through a mixing coil before reaching the dialysis unit. In place of a dialysis membrane, a Gelman 1.2- $\mu \mathrm{m}$ Metricel membrane filter is used. This filter is sandwiched between the two dialyzer plates, and the bloodperfusate mixture is passed on one side of the membrane. The filtered plasma-perfusate mixture is extracted from the opposite side of the membrane at a rate of $6 \mathrm{ml} / \mathrm{min}$. Red cells do not pass through the filter intact. For each passage through the dialysis unit, one-half of the old plasma (3 $\mathrm{ml} / \mathrm{min}$ ) is extracted and $3 \mathrm{ml} / \mathrm{min}$ of new perfusate is added in its place. This filtrate is set aside for subsequent biochemical assays and the rejuvenated blood returned to the oxygenator-reservoir.

\section{The Perfusate}

Any suitable perfusate must contain physiologic levels of essential electrolytes and must be osmotically similar to plasma. The basic perfusate used in this preparation consists of a balanced electrolyte solution to which bovine serum albumin (Cohn, Fraction V, Sigma Chemical Co. St. Louis, Mo.) is added to a final concentration of $4.0 \mathrm{~g} / \mathrm{dl}$. Glucose is added to a concentration of $150 \mathrm{mg} / \mathrm{dl}$ and the final solution is adjusted to an osmolality of $315 \mathrm{mosm} / \mathrm{liter}$ by adding $12.5 \%$ mannitol as necessary. One thousand units of aqueous heparin (heparin sodium injection, 1000 units/ml, Upjohn Company, Kalamazoo, Mich.) is added to 
the approximately $400 \mathrm{ml}$ of perfusate to prevent blood clotting. In its usable form the perfusate contains the following electrolyte concentrations: sodium, 127 meq/liter; chloride, $105 \mathrm{meq} /$ liter; bicarbonate, 40 meq-liter; calcium, 5 meq/liter; potassium, $3.5 \mathrm{meq} / \mathrm{liter}$; magnesium, 2 meq/liter; phosphate, 2 meq/liter; and sulfate, $1 \mathrm{meq} /$ liter. $\mathrm{pH}$ is adjusted to 7.4 with $1.0 \mathrm{M} \mathrm{NaOH}$.

\section{Organ Isolation Procedure}

Pancreaticoduodenal preparations were obtained initially by our group [10] using the technique of Ritchie and Hardcastle [22]. In order to eliminate problems with cannulation of the splenic artery branches (vessels with luminal diameters of 1.5 to $1.8 \mathrm{~mm}$ ), a simplified preparation was developed. This modified isolation technique is presented in the following paragraphs.

Conditioned mongrel dogs weighing 20 to $25 \mathrm{~kg}$ are anesthetized with Suritol, intubated, and maintained on a Harvard respirator. The abdomen is opened and after ligation of the left gastric artery close to its origin from the celiac axis, the stomach is removed. The spleen is removed after ligating its vessels within the hilum. This preserves circulation to the tail of the pancreas. The abdominal aorta is isolated from the level of the renal arteries to the diaphragm. Visceral branches to the adrenal, kidney, and liver are individually ligated and divided. The superior mesenteric artery is ligated and divided distal to the origin of the inferior pancreaticoduodenal artery. The entire small and large intestine are then removed from the abdominal cavity.

An 8- to $10-\mathrm{cm}$ juxtapancreatic segment of duodenum is preserved and cannulated proximally and distally to allow for perfusion and collection of duodenal secretions. A $3-\mathrm{cm}$ incision is made along the antimesenteric border of the duodenum and the major pancreatic duct is identified with a fine silver probe. The extraduodenal portion of the pancreatic duct is isolated, cannulated with a fine polyethylene catheter, and divided. Care is taken to identify accessory pancreatic ducts which when found are ligated. The latter procedure effectively dissociates the pancreatic duct from duodenal drainage. Aqueous heparin sodium (4 mg/ $\mathrm{kg}$ ) is administered intravenously and is allowed to circulate. The infrapancreatic segment of aorta is then divided between noncrushing clamps and cannulated with a 1.5$\mathrm{cm}$ i.d. stainless-steel perfusion cannula. The aorta is divided proximally at the level of the diaphragm and a closed-end pressure monitoring cannula is inserted. Pump flow is initiated with perfusate not containing autologous red blood cells at a rate of 20 $\mathrm{ml} / \mathrm{min}$. The $\mathrm{dog}$ is rapidly exsanguinated into blood collection bags containing citratephosphate-dextrose (CPD, Travenol Corp., Deerfield, Ill.). These autologous red blood cells are washed free of citrate and serum prior to their addition to the perfusion medium. The organ preparation is purged of microcirculatory debris by maintaining perfusion without red blood cells for $2 \mathrm{~min}$. The portal vein is isolated between ligatures. Perfusion is arrested temporarily and a 1.5$\mathrm{cm}$ i.d. stainless-steel collection cannula is placed in the juxtapancreatic segment of the portal vein. Care is taken not to compromise venous outflow from the superior pancreaticoduodenal vein. The final preparation is illustrated in Fig. 2. Circulatory arrest time through the isolated organ preparation averages 30 seconds. The organ preparation is then transferred to the perfusion apparatus and allowed to equilibrate for $30 \mathrm{~min}$ under basal conditions.

The system is primed with $300 \mathrm{ml}$ of perfusate mixture and autologous red blood cells are added to a final hemoglobin concentration to 7.0 to $10 \mathrm{~g} / \mathrm{dl}$. This usually results in a total perfusate volume of 350 to $400 \mathrm{ml}$. Arterial and venous blood gas determinations are performed on a Radiometer Copenhagen ABL2C gas analyzer. Glucose is measured by the oxygen rate method on a Beckman glucose/BUN analyzer. Sodium and potassium are analyzed either by flame emission (IL 343 digital 


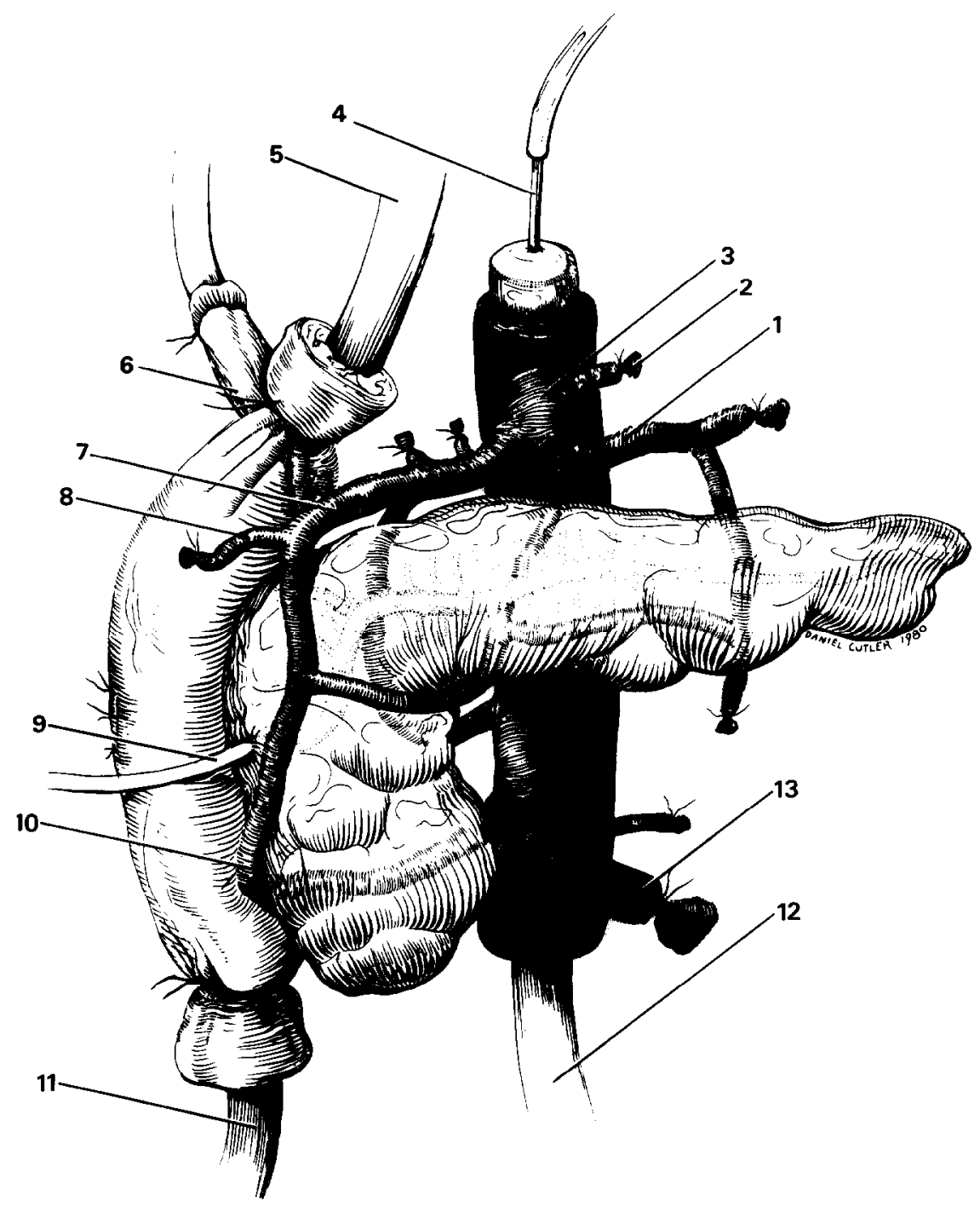

FIG. 2. Illustration of anatomical relationships of the final, isolated pancreaticoduodenal preparation. The labeled structures are as follows: (1) splenic artery, (2) left gastric artery, (3) celiac trunk, (4) aortic pressure monitoring cannula, (5) perfusion tubin proximal duodenum, (6) portal vein, (7) superior pancreaticoduodenal artery, (8) right gastroepiploic artery, (9) pancreatic duct cannula, (10) inferior pancreaticoduodenal artery, (11) collection tubing in distal duodenum, (12) aortic perfusion cannula.

flame photometer) or by potentiometric determination using ion-selective electrodes (Beckman Astra-4). Chloride is determined by colormetric titration using a silver chloride electrode and silver chloride anode (Beckman Astra-4). Osmometry is determined by freezing point depression (Osmette A Model 5002, Precision Systems Inc.). Calcium is determined on an Electro-
Nucleonics Gemini using a cresolphthaleincomplexone binding technique. Arterial and venous pressures are monitored and charted continuously on a Hewlett-Packard $\mathbf{8 8 0 0}$ medical system employing C8805B pressure amplifiers and $1280 \mathrm{~B} / \mathrm{C}$ pressure transducers. All biochemical and acid-base parameters are assessed following a 30min equilibration period and hourly there- 
after. The duodenal segment is perfused at a constant rate with lactated Ringer's solution containing $0.6 \%$ phenol red indicator. Change from acid orange to purple suggests contamination of the duodenal effluent by alkaline pancreatic exocrine secretions.

Biopsy specimens from the body of the in situ- and ex vivo-perfused pancreas are obtained at hourly intervals. Specimens for light microscopy are fixed in $10 \%$ buffered neutral formalin, paraffin processed, and stained with hematoxylin and eosin. Specimens for electron microscopy are fixed in $2.53 \%$ paraformalin/glutaraldehyde, postfixed in $1 \%$ osmium containing $0.05 \mathrm{M}$ potassium ferricyanide for $60 \mathrm{~min}$ and stained in $1 \%$ uranil acetate prior to being embedded in Epon. Gold and silver vaporcovered sections are stained with uranil acetate and lead citrate and examined using a Phillips 400 electron microscope.

\section{RESULTS}

The following criteria were used to assess viability of the organ preparation throughout the perfusion period: gross and histologic appearance of the gland, constancy of oxygen consumption, vascular resistance, pancreatic exocrine response to stimulation, and contractility of the duodenal segment.

\section{Perfusion Characteristics}

The wet weight of the entire pancreaticoduodenal block varied from 90 to $115 \mathrm{~g}$ with a mean pancreas weight of approximately $50 \mathrm{~g}$. Arterial perfusion was maintained constant at $20 \mathrm{ml} / \mathrm{min}$ to achieve venous oxygen saturations of 65 to $75 \%$ at $37^{\circ} \mathrm{C}$ under resting conditions. Oxygen consumption was calculated using the formula:

oxygen extraction $\left(\mathrm{ml}\right.$ of $\mathrm{O}_{2} / \mathrm{ml}$ of flow $/ \mathrm{min}$

$$
\begin{gathered}
\begin{array}{c}
1.34 \times \text { hgb (Art Sat }- \text { Ven Sat }) \\
+0.003(\mathrm{pa} 02-\mathrm{Pv} 02)
\end{array} \\
\text { flow rate }(\mathrm{ml} / \mathrm{min})
\end{gathered}
$$

Oxygen extraction in six experimental dogs remained constant throughout the perfusion period and averaged $0.05 \pm 0.02 \mathrm{ml}$ $\mathrm{O}_{2}$ per milliliter of blood flow per minute. Oxygen consumption appeared to be a reliable measure of organ viability. Poisoning the gland with $0.06 \mathrm{~g}$ sodium cyanide $(\mathrm{NaCN})$ resulted in a significant $(P<0.001)$ reduction in oxygen extraction to 0.006 $\pm .0003 \mathrm{ml} \mathrm{O}_{2}$ per milliliter blood flow per minute.

Several investigators $[1,4,22]$ have observed arterial perfusion pressures of 90 to $110 \mathrm{~mm} \mathrm{Hg}$ immediately after the initiation of perfusion. Arterial pressure usually declined in our study during the equilibration period to a final constant level of 30 to $50 \mathrm{~mm} \mathrm{Hg}$. We observed that initial arterial pressure accurately reflected the eventual outcome of the perfusion. Many preparations with initial pressures of greater than 60 to $75 \mathrm{~mm} \mathrm{Hg}$ failed to recover and developed progressive gross evidence of pancreatitis. Pathologic examination of these organs routinely demonstrated disseminated microvascular thrombosis. In common with all such events was a prolonged period of organ stasis during which perfusion was arrested in order to insert the portal venous cannula. Stasis problems were entirely eliminated by flushing the organ with perfusate mixture as previously described.

\section{Gross and Histologic Appearance}

The gross appearance of the preparation usually remained normal throughout the period of perfusion. Spotty, petechial hemorrhages on the surface of the gland were observed frequently but appeared to be of no functional or pathological significance. Edema of the gland was a constant occurrence but in general was negligible with perfusions of $4 \mathrm{hr}$ or less. Leakage of blood from the preparation was minimal throughout the perfusion period and averaged less than $0.5 \mathrm{ml} / \mathrm{min}$. Significant bleeding was generally associated with inadequate hemostasis. Viability of the duodenal 


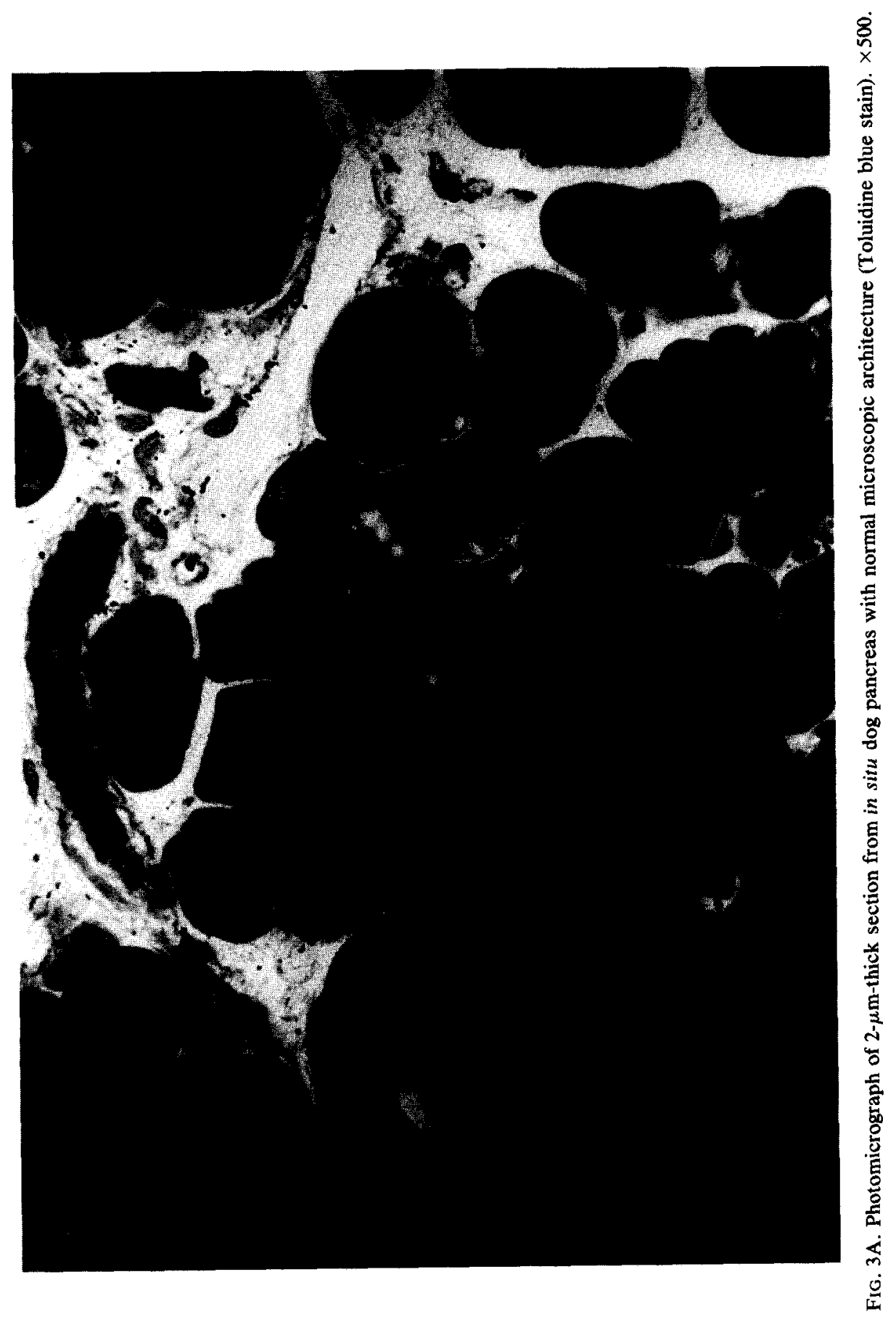




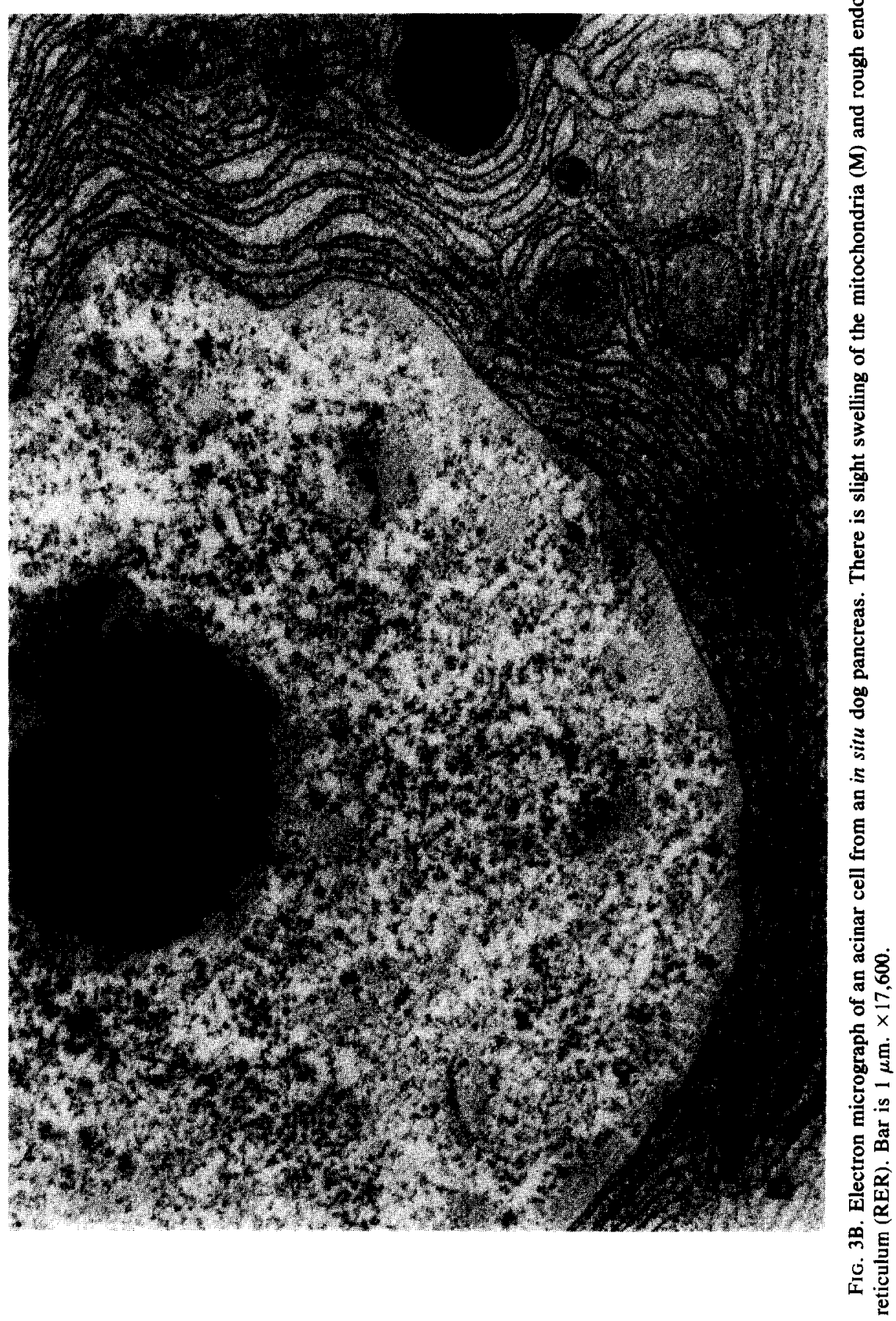

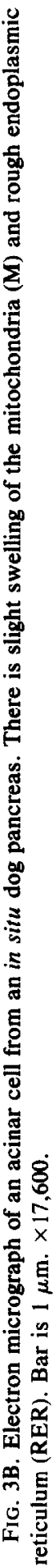




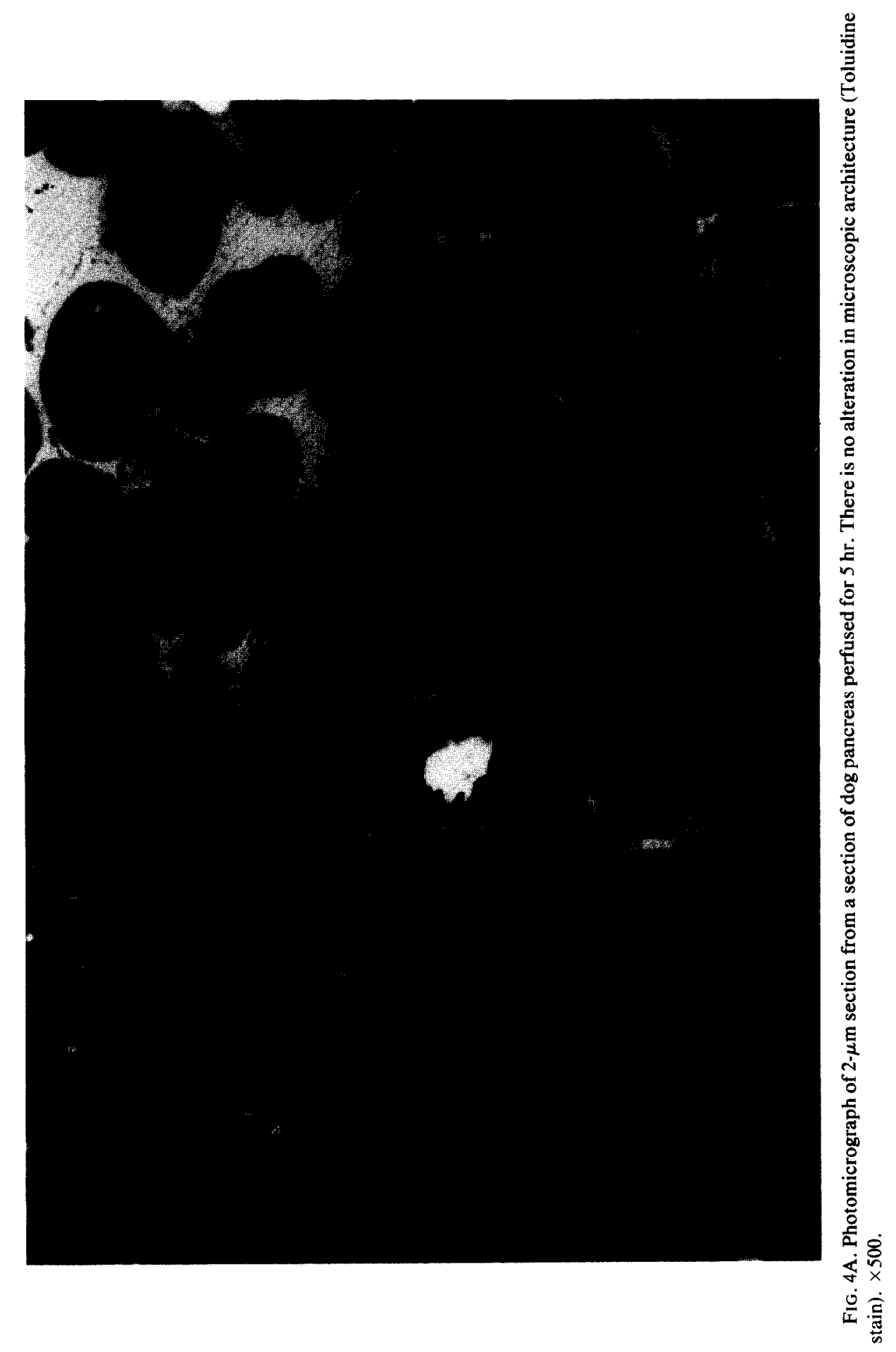




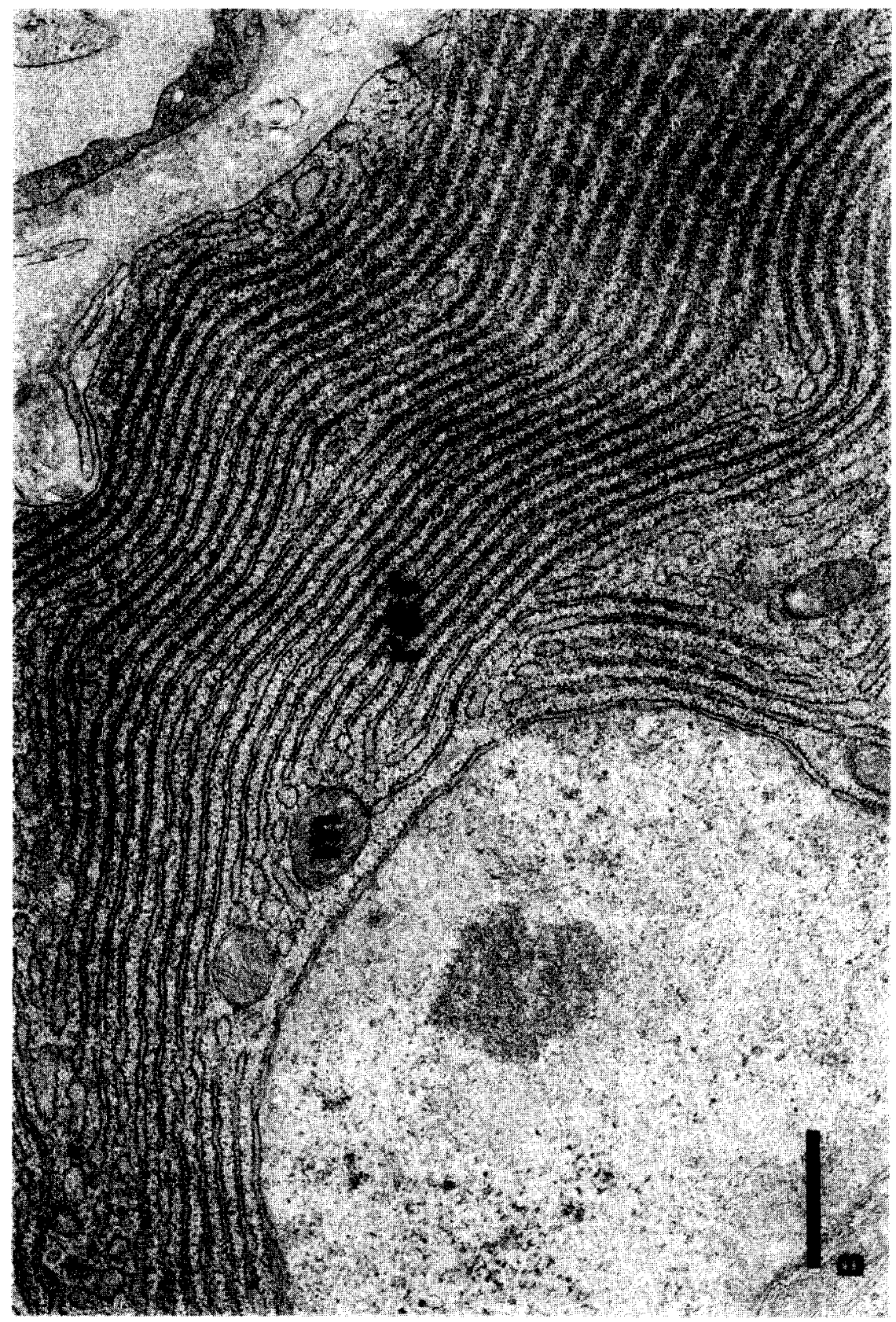

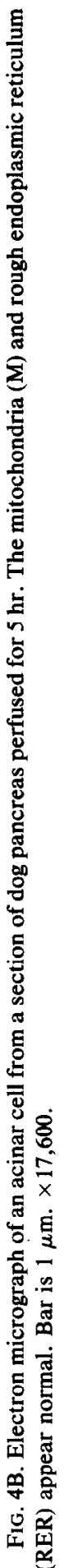


segment was assessed by observing contractility. Duodenal contractions became less forceful with prolonged perfusion and were rarely vigorous in perfusions exceeding $4 \mathrm{hr}$.

Light microscopy demonstrated minimal variation in control and perfused pancreas specimens. There was no observable alteration in acinar pattern, nuclei, or cytoplasm. The ultrastructural findings of in situ biopsies obtained at hourly intervals during perfusion showed slight swelling of the mitochondria and rough endoplasmic reticulum. Golgi complexes appeared normal and there were normal stores of zymogen granules. Chromatin patterns were unremarkable. Specimens examined at hourly intervals during ex vivo perfusion showed similar findings. The mitochondria and rough endoplasmic reticulum as well as the golgi complexes and stores of zymogen granules were unremarkable. No autophagocytic changes were observed during any perfusion period of $4 \mathrm{hr}$ or less. Figs. $3 \mathrm{~A}$ and $B$ are light and electron micrographs, respectively, of an in situ dog pancreas. Figures $4 \mathrm{~A}$ and $4 \mathrm{~B}$ are light and electron micrographs of dog pancreas biopsies obtained after a 5-hr period of ex vivo perfusion. There is remarkably little variation in either light microscopic or ultrastructural appearance between the two biopsy specimens.

\section{Exocrine Responses to Stimulation}

Table 1 summarizes our experience with basal- and secretin-stimulated pancreatic exocrine function in two ex vivo preparations. Resting flow of clear pancreatic juice was approximately $0.035 \mathrm{ml} / \mathrm{min}$. Pancreatic exocrine responses were similar in ex vivoperfused specimens to which heparin sodium had been added and in nonheparinized in situ preparations. This suggests that heparin per se is not a significant pancreatic exocrine secretagogue. In response to a constant reservoir infusion of 3 units/min of Boots
TABLE 1

EXocrine FunCtION OF THE EX VIVO, HEMOPERFUSED Pancreas Preparation

\begin{tabular}{|c|c|c|c|}
\hline $\begin{array}{l}\text { Pancreatic } \\
\text { exocrine } \\
\text { secretion }\end{array}$ & Basal & $\begin{array}{l}\text { Average values } \\
30 \text {-min after } \\
\text { initiation of con- } \\
\text { tinuous secretin } \\
\text { infusion } \\
(3 \text { units/min })^{a}\end{array}$ & $\begin{array}{l}\text { Average values } \\
\text { 30-min following } \\
\text { discontinuation of } \\
\text { secretin infusion }{ }^{a}\end{array}$ \\
\hline Volume (ml) & 1.95 & 14.50 & 5.9 \\
\hline Rate (ml/min) & 0.035 & 0.285 & 0.180 \\
\hline $\begin{array}{l}\text { Protein }(g / d) \\
\text { Amylase }\end{array}$ & 1.06 & 0.66 & 0.49 \\
\hline$\left(\mu \mathrm{g} / \mathrm{ml} \times 10^{3}\right)$ & 575 & 439 & 270 \\
\hline Lipase & & & \\
\hline$\left(\mu \mathrm{g} / \mathrm{ml} \times 10^{3}\right)$ & 6.2 & 3.52 & 2.85 \\
\hline $\mathrm{Cl}^{-}(\mathrm{meq} / \mathrm{dl})$ & 96 & 58 & 74 \\
\hline $\mathrm{HCO}_{3-}(\mathrm{meq} / \mathrm{dl})$ & 63 & 110 & 105 \\
\hline
\end{tabular}

secretin (Boots Co. Ltd., Nottingham, England), the rate of pancreatic juice flow increased nearly 10 -fold from 0.035 to 0.28 $\mathrm{ml} / \mathrm{min}$. This response lasted throughout the infusion period and was associated with decrease in arterial pressure and venous oxygen saturation. Concentrations of protein, amylase, lipase, and chloride in pancreatic juice decreased while that of bicarbonate rose nearly $75 \%$ to a maximal level of $110 \mathrm{meq} / \mathrm{liter}$ after $30 \mathrm{~min}$. Cessation of secretin infusion was associated with normalization toward baseline values.

Electrolyte and acid-base balance remained within physiologic ranges throughout the period of perfusion. There was a slight tendency following secretin stimulation toward acidosis (mean change in $\mathrm{pH}$ of 0.14 units); however, this was easily adjusted by adding sodium bicarbonate at intervals whenever necessary.

The introduction of in-line dialysis facilitated electrolyte and acid-base maintenance. The percentage loss per hour of amylase into the dialysis was calculated according to the formula:

amount amylase measured

in dialysate/hour

total amylase measured in system ${ }^{1}$

${ }^{1}$ Amount loss plus that remaining. 
In the unstimulated or basal state, amylase was cleared from the system at a rate of $33 \pm 12 \% / \mathrm{hr}$. No significant change in amylase clearance was observed after secretin stimulation $(25 \pm 3 \% / \mathrm{hr}$, PNS $)$. Similar calculations of lipase clearance demonstrated an average basal cumulative loss of $35 \pm 27 \% / \mathrm{hr}$ which did not change significantly after secretin stimulation.

Hemolysis is a constant sequela of perfusion systems which incorporate pumping mechanisms. Serum-free hemoglobin was measured in the present study using a spectrophotometric method. Normal values range from 4 to $40 \mathrm{mg} / \mathrm{dl}$. Although the average hourly rate of production of free hemoglobin remained constant at $4.8 \pm 1.1$ $\mathrm{mg} / \mathrm{dl} / \mathrm{hr}$, serum levels of free hemoglobin increased from a baseline value of 4.95 $\pm 2.2 \mathrm{mg} / \mathrm{dl}$ to $24.0 \pm 16.6 \mathrm{mg} / \mathrm{dl}$ after $4 \mathrm{hr}$ of perfusion. These data suggest effective but incomplete filtration and elimination of free hemoglobin. The variable light pink to cherry red appearance of dialysate media correlated grossly with serum levels of free hemoglobin.

\section{DISCUSSION}

Perfusion has been defined by Ross as "the maintenance of an organ in a viable state, isolated from the animal by means of the mechanically assisted circulation of an artificial medium through its vascular bed" [14]. In contrast to standard in vitro techniques including tissue homogenates, slices, and cellular fractions, organ perfusion is complex and its use requires some justification.

Perfused organ preparations are suitable for the study of enzyme-mediated anabolic and catabolic activities. Alternative techniques are disadvantaged by virtue of their artificiality. Hems et al. demonstrated that the rate of gluconeogenesis in the perfused rate liver preparation approximated that observed in vivo, and in fact was considerably higher than that observed in liver slice or tissue homogenate preparations [13]. Compartmentalization is maintained in wholeorgan preparations. Division into cellular, extracellular, vascular, interstitial, and in our case luminal compartments, is necessary for integrated organ homeostasis and in addition appears to have significant regulatory importance.

The general principles for experimental perfusion include: (1) an organ with a distinct vascular bed that can be isolated from contiguous organs; (2) a perfusion medium that is separate from the perfused organ and is also an appropriate carrier of oxygen; (3) a means of circulating the perfusion medium in order to provide oxygen for respiratory purposes and nutrient substrates for metabolic purposes; and (4) a means of providing oxygen as well as a controlled environment.

Once-through perfusion systems are simplistic and obviate the need for an oxygenator or pumping mechanism. Their investigational applications, however, are limited by the need for a large volume of perfusion medium and the fact that the rate of nutrient uptake for organ metabolism is highly flow dependent. Recirculation perfusion preparations, on the other hand, are disadvantaged by several factors. Mechanical pumps are necessary to maintain perfusion. Trauma to red blood cells is an unavoidable sequela of this technique and results in the production of free hemoglobin that is potentially deleterious to organ homeostasis. Metabolic activity results in the depletion of glucose and other nutrient substrates. Potentially noxious organic acids, most notably lactate, are produced. Finally, proper oxygenation of the isolated organ requires an air-liquid (oxygenblood) interface which may result in progressive dehydration of the blood.

All of the aforementioned difficulties can be minimized by employing a once-through perfusion system. Our experience with a once-through perfusion-red cell recoveryreperfusion system was uniformly poor. Red cells recovered from the venous drain- 
age were washed in isotonic saline, resuspended in perfusion medium, and added to the reservoir for recycling. In spite of efforts to adjust toxicity of the perfusion medium (up to $400 \mathrm{mosm} / \mathrm{liter}$ ) with $12.5 \%$ mannitol, unexplained edema was a constant observation.

The introduction of a filtration mechanism or artificial kidney provides a mechanism to selectively remove noxious substances resulting from organ metabolism and pump trauma to red blood cells. The constant introduction of new perfusate avoids "tissue-medium" equilibration problems alluded to by Ross [24] with oncethrough perfusion systems, and facilitates maintenance of a constant electrolyte and acid-base environment.

Many types of perfusion media, including homologous fresh or stored blood or semisynthetic products either alone or in combination, have been used with varying results [2, 24]. Hechter et al. [12] and more recently Kowaleski and Kolodej [15] have reported successful organ perfusion omitting blood from the medium. The latter investigators have used liquid fluorocarbon composed primarily of fluorochemical FC47 with pluronic F-68 added as the surfactant [15]. Although this artificial blood substitute has proved satisfactory to study ex vivo pancreatic exocrine function, for inexplicable reasons it has proved unsatisfactory for the study of gastric acid secretory function. We, like Miller, feel that physiologic responses of an ex vivo recirculation-perfused organ can be best studied "under physical conditions most closely approximating those in the intact animal" [18]. For this reason, washed red blood cells in a semisynthetic medium are now used exclusively.

Several subtle points regarding perfusion media deserve emphasis. Oxygen transport may be accomplished by several techniques, the simplest of which in our ex- perience is as oxyhemoglobin bound to erythrocytes. Whole blood must be defibrinated or anticoagulated with heparin or CPD solution prior to use. Autologous or homologous blood may be used fresh or up to 1 week after collection [21]. The use of this medium may be limited due to the large volumes of blood necessary for oncethrough perfusion or large-volume recirculation perfusion systems.

Plasma expanders must be added to any synthetic perfusion medium to maintain colloid osmotic pressure and prevent fluid loss from the vascular compartment. A variety of plasma substitutes have been used, including bovine albumin (Cohn Fraction V), dextran, polyvinylpyrrolidone (PVP), and gelatin.

Albumin would appear to be the most natural colloid substitute and crystalline bovine albumin (Cohn Fraction V), a product of alcohol fractionation of total plasma protein, has found wide application in perfusion media. Albumin concentrations of 3 to 5 g/dl perfusate are satisfactory for most organ perfusions, but higher concentrations (up to $7 \mathrm{~g} / \mathrm{dl}$ ) have been used in high-pressure, arterial perfusions to prevent edema formation [25]. Concentrations of $4 \mathrm{~g} / \mathrm{dl}$ proved satisfactory in our pancreas perfusion studies.

The overenthusiastic use of bovine albumin must be tempered by a foreknowledge of its disadvantages. When added to a buffer medium, albumin may affect oxygen solubility and displace bicarbonate resulting in unpredictable variation in $\mathrm{pH}$. Further, the addition of albumin to any perfusion medium may decrease the concentration of ionized calcium. The latter may have some important disadvantages in isolated heart perfusion, but has not proved to be a significant problem in our pancreas perfusion studies. Batches of bovine albumin vary in composition and some preparations may be toxic in organ perfusions. Preperfusion 
defatting and dialysis of bovine albumin is advocated by some authors to remove longchain fatty acids and small molecular contaminants [5]. In our experience, contaminated bovine albumin emits a peculiar hydrocarbon-like odor. We recommend limiting the use of one "known" batch of albumin to any single series of experiments and returning immediately to the manufacturer any batch that has an unusual odor. Fortunately, quality control in manufacture is sufficient to limit the occurrence of this problem.

A pumping mechanism is requisite to most recirculation perfusion systems. Its use may be obviated, as previously discussed, in hydrostatic or once-through perfusion models. Many impeller pumps are commercially available, including steady and pulsatile flow types. Studies on oxygen consumption or glucose metabolism using both varieties in several isolated organ perfusion systems have failed to demonstrate any significant differences [23]. Our selection of an open-valve, pulsatile pump (Harvard Instruments Model 1405) was based on Mortimer's observations that "open" pumps result in less red blood cell trauma and hemolysis in comparison with "closed" pumps (e.g., Ambec and WatsonMarlow roller pumps) [19]. Valid requirements of any pump should include durability, accuracy, and output capacity that exceeds the maximum anticipated flow requirements of the isolated organ preparation.

Isolated perfusion preparations are sophisticated tools for investigating physiologic and exocrine secretory functions of the pancreas; excellent overviews by Nardi et al. in 1963 [20] and Herman-Taylor in 1968 [14] have appeared in the literature. Our studies are compatible with earlier observations by Sussman et al. that normalcy and constancy of oxygen consumption and vascular resistance are the most valuable criteria of organ viability [27].
Physical appearance of the preparation appear to be the least reliable sign of viability.

Recirculation perfusion of the dog pancreas may have limited application in studies of the enteroinsular axis. These limitations appear to be imposed by the closedloop nature of the recirculation apparatus. Several investigators have demonstrated release of immunoreactive peptides (hormones) into both gastric [29] and pancreatic juice $[10,17]$. Whereas the dynamics of hormone release into pancreatic juice are easy to quantitate, interpretation of release patterns into blood is considerably more difficult. In a previous ex vivo study of immunoreactive gastrin release into pancreatic juice and portal blood in the dog, we reported an average dialysate loss of total gastrin in the system of $7.8 \%$ every $30 \mathrm{~min}$. This necessitated upward correction of each portal vein gastrin value by a factor of $0.03 \%$. The inaccuracies implicit in this type of system may prove to be prohibitive. The lack of an efficient clearance mechanism will invariably lead to accumulation over time of immunoreactive peptides; heightened blood levels may in turn alter servomechanisms which under normal conditions regulate the patterns and quantities of hormones released into juice and blood. These problems can be minimized by the use of a once-through perfusion preparation. However, use of the large volumes of perfusate necessary for once-through perfusion may eventuate in problems such as blood stasis and disequilibrium of tissue and perfusate. Similarly the large volumes of medium required may preclude the use of autologous blood. Washed red blood cells in a semisynthetic medium may prove to be a rational alternative to autologous blood in this setting. Ex vivo perfusion has proved to be a valuable investigational tool for studies of pancreatic physiology and exocrine secretory function. The potential for additional, endocrine-related studies, how- 
ever, is plagued by limitations imposed by available technology.

\section{CONCLUSIONS}

The ex vivo, hemoperfused preparation is a suitable model to study pancreatic physiology and exocrine secretory function in the dog. Changes in vascular resistance and oxygen consumption over time are the most useful criteria of organ viability. Gross appearance is of limited value. Incorporation of a modified dialysis unit into the perfusion system facilitates maintenance of acid base and electrolyte balance as well as glucose homeostasis. A mechanism is provided for elimination from the system of substances which are potentially deleterious to the preparation. Endocrinerelated studies, including the dynamics of hormone release into pancreatic juice and blood, are complicated by a recirculation perfusion system.

\section{REFERENCES}

1. Abraham, R., Dawson, W., Grasso, P., and Goldberg, L. Lysosomal changes associated with hyperoxia in the isolated perfused rat liver. Exp. Mol. Pathol. 8: 370, 1968.

2. Andjus, R. U., Suhara, K., and Sloviter, H. A. An isolated perfused rat brain preparation, its spontaneous and stimulated activity. J. Appl. Physiol. 22: $1033,1967$.

3. Babkin, B. P., and Starling, E. H. A method for the study of the perfused pancreas. J. Physiol. 61: 245, 1926.

4. Case, R. M., Harper, A. A., and Scratcherd, T. Water and electrolyte secretion by the perfused pancreas of the cat. J. Physiol. 196: 133, 1968.

5. Chen, R. F. Removal of fatty acids from serum albumin by charcoal treatment. J. Biol. Chem. 242: $173,1967$.

6. Creutzfeldt, W., Ebert, R., Arnold, R., Frerichs, H., and Brown, J. C. Gastric inhibitory peptide (GIP), gastrin and insulin response to test meal in celiac disease and after pancreatectomy. Diabetologia 12: 279, 1976.

7. Ebert, R., Creutzfeldt, W., Brown, J. C., Frerichs, H., and Arnold, R. Response of GIP to test meal in chronic pancreatitis; relationship to endocrine and exocrine insufficiency. Diabetologia 12: 609 , 1976.

8. Ebert, R., Frerichs, H., and Creutzfeldt, W. Serum GIP response in maturity-onset diabetes and in juvenile diabetes. Diabetologia 12(A): 388, 1976.

9. Ebert, R., Williams, B., Brown, J. C., and Creutzfeldt, W. Serum GIP levels in obese patients and after weight reduction. Eur. J. Clin. Invest. 6(A): 327, 1976.

10. Eckhauser, F. E., Vinik, A. I., McLeod, M., Porter-Fink, V., and Fiddian-Green, R. Secretin, a stimulus for duodenal and pancreatic gastrin release: Possible pathogenetic significance in Zollinger-Ellison syndrome. J. Surg. Res. 28: $356,1980$.

11. Geriche, J. E., Charles, M. A., and Grodsky, G. M. Characterization of the effects of arginine and glucose on glucagon and insulin release from the perfused rat pancreas. J. Clin. Invest. 54: 833, 1974.

12. Hechter, O., Zaffaroni, A., Jacobsen, R. P., Levy, H., Jeanloz, R. W., Schenker, V., and Pincus, G. The nature and biogenesis of the adrenal secretory product. Rec. Progr. Horm. Res. 6: 215, 1951.

13. Hems, R., Ross, B. D., Berry, M. N., and Krebs, H. A. Gluconeogensis in the perfused rat liver. Biochem. J. 101: 284, 1966.

14. Herman-Taylor, J. A technique for perfusion of the isolated canine pancreas; responses to secretin and gastrin. Gastroenterology 55: 488, 1968.

15. Kowaleski, K., and Kolodej, A. Secretory function of the isolated canine pancreas perfused with fluorocarbon emulsion. Surg. Gynecol. Obstet. 146: 375, 1978.

16. Long, J. A., and Lyons, W. R. A small perfusion apparatus for the study of surviving isolated organs. J. Lab. Clin. Med. 44: 614, 1954.

17. McLeod, M., Eckhauser, F. E., Glaser, B., PorterFink, V., and Vinik, A. I. Evidence for dissociation of the release of pancreatic polypeptide (PP) into blood and pancreatic juice in the dog. (In preparation.)

18. Miller, L. L. Some direct actions of insulin, glucagon and hydrocortisone on the isolated perfused rat liver. Rec. Prog. Horm. Res. 17: $539,1961$.

19. Mortimer, G. B. Effect of insulin on potassium transfer in isolated rat liver. Amer. J. Physiol. 200: 1315, 1961.

20. Nardi, G. L., Greeps, J. M., Chambers, D. A., McRae, C. E., and Skinner, D. B. Physiologic perigrinations in pancreatic perfusion. Ann. Surg. 158: 830, 1963.

21. Richardson, G. S., and Ulfelder, H. Experiences 
with a simplified method of small organ perfusion. Surgery 48: 237, 1960.

22. Ritchie, H. D., and Hardcastle, J. D. The isolated perfused canine pancreas. In H. D. Cooper and J. D. Castle (Eds.), Isolated Organ Perfusion. Baltimore; Univ. Park Press, 1973. P. 170.

23. Ritter, E. R. Pressure/flow relations in the kidney: Alleged effects of pulse pressure. Amer.J. Physiol. 168: 480, 1952.

24. Ross, B. D. Perfusion Techniques In Biochemistry. London: Oxford Univ. Press, 1972.

25. Ruderman, N. B., Houghton, C. R. S., and Hems, R. Evaluation of the isolated perfused rat hind-quarter for the study of muscle metabolism. Biochem. J. 124: 639, 1971.

26. Steer, M. L., Glazer, G., and Manabe, T. Direct effects of ethanol on exocrine secretion from the in vivo rabbit pancreas. Dig. Dis, Sci. 24: 769, 1979.

27. Sussman, M. L., Vaughan, G. D., and Timmir, R. F. An in vitro method for studying insulin secretin in the perfused isolated rat pancreas. Metabolism 15: 466, 1968.

28. Toledo-Pereyra, L. H., Valgee, K. D., Castellanos, J., and Chee, M. Hypothermic pulsatile perfusion: Its use in the preservation of pancreases for 24 to 48 hours before islet cell transplantation. Arch. Surg. 115: 95, 1980.

29. Uvnas-Wallensten, K., and Rehfeld, J. F. Molecular forms of gastrin in antral mucosa, plasma and gastric juice during vagal stimulation in anesthetized cats. Acta Physiol. Scand. 98: 217, 1976. 